\title{
Isolated Focal Infantile Hemangioma of the Alveolar Ridge with Glucose Transporter-1 Reactivity: An Aberrant Presentation
}

\author{
Divya Natarajan ${ }^{1}$, Shanmugam Muthukali ${ }^{2}$, Nachiammai Nachiappan ${ }^{3}$, Joe Louis Chiriyamkandath ${ }^{4}$, Jayanthi Kannappan ${ }^{5}$
}

\begin{abstract}
Aim and objective: To give dental practitioners an insight into the unusual presentation of focal infantile hemangioma on the alveolar ridge showing immunoreactivity to glucose transporter-1 (GLT1). We also recommend the importance of using standardized terminologies to describe the type of hemangioma.

Background: One of the most commonly seen and misdiagnosed vascular tumors of the skin and soft tissues of the head and neck region in children are infantile hemangiomas. Infantile hemangiomas developing at extracutaneous locations such as the oral cavity are rare, especially without cutaneous involvement.

Case description: A 9-month-old infant girl presented to the Pediatric Dental Department with a swelling over the maxillary alveolar ridge. The solitary exophytic mass appeared after birth and gradually enlarged in size with episodes of bleeding during feeding. Clinical examination revealed a reddish-pink pedunculated mass that bled profusely on palpation. The lesion was excised by electrocautery and was found to be express glucose transporter-1 confirming the diagnosis of infantile hemangioma. The patient was followed up for 1 year and showed no recurrence.

Conclusion: Hemangiomas are usually misinterpreted to be pyogenic granulomas, vascular malformation, reactive granulation tissue, or congenital granular cell tumor. This report highlights the use of glucose transporter-1 when there is suspicion of hemangioma for immunohistochemical distinction from other histologically similar vascular lesions of the oral cavity.

Clinical significance: Dental surgeons treating vascular lesions of the oral cavity should advocate for surgical management techniques that also produce hemostasis due to the risk of intraoperative bleeding.

Keywords: Alveolar ridge, Benign tumor, Glucose transporter-1, Hemangioma, Infant, Infantile hemangioma, Oral pathology, Vascular tumor. International Journal of Clinical Pediatric Dentistry (2021): 10.5005/jp-journals-10005-1923
\end{abstract}

\section{BACKGROUND}

Hemangiomas are benign vascular tumors that may be the result of ectasia of preexisting vessels, a combination of ectasia and new growth, or new growth alone. ${ }^{1}$ The incidence varies from 1 to $2.6 \%$ up to $10 \%$ in the first year of life, with a higher female preponderance (ratio of 3:1). ${ }^{2-4}$ Among the Indian population, the incidence was reported to be $12.7 \%$ with a male to female ratio of 1:1.4 in preterm infants. ${ }^{5}$ They present more frequently in premature infants with $<1,000 \mathrm{~g}$ birthweight, firstlings, whites, paternal age $<35$ years, and infant born to mothers of higher maternal age complicated by hyperemesis, multiple gestations, placenta previa, preeclampsia, and chorionic-villus sampling., $3,6,7$

The most common site of infantile hemangiomas $(\mathrm{IHs})$ is the craniofacial region (60\%), followed by trunk (25\%) and extremities (15\%); and $80 \%$ of them are solitary in nature. ${ }^{8}$ It presents on the ventral surface of the tongue, lower lip, buccal mucosa, upper lip, and palatal mucosa. ${ }^{2,9,10}$ Its occurrence on the alveolar ridge is rare in children.

Numerous terms are used indiscriminately to designate the vast subtypes of hemangiomas by clinicians and in the literature causing difficulty in diagnosis and management. Histopathological assessment is subjective and cannot render a correct diagnosis of hemangiomas. ${ }^{3}$ Hence, a formulated workup using this immunohistochemical marker and diagnosis using standard terminology is a must for the management of hemangiomas to avoid misinterpretation and reclassification of vascular lesions. This immunohistochemical biomarker GLUT1 has been proven to
1,4,5 Department of Pediatric Dentistry, Chettinad Dental College and Research Institute, Kancheepuram, Tamil Nadu, India

${ }^{2}$ Department of Periodontics, Chettinad Dental College and Research Institute, Kancheepuram, Tamil Nadu, India

${ }^{3}$ Department of Oral Pathology, Chettinad Dental College and Research Institute, Kancheepuram, Tamil Nadu, India

Corresponding Author: Divya Natarajan, Department of Pediatric Dentistry, Chettinad Dental College and Research Institute, Kancheepuram, Tamil Nadu, India, Phone: +91 9790774858, e-mail: dr.divya@outlook.in

How to cite this article: Natarajan D, Muthukali S, Nachiappan N, et al. Isolated Focal Infantile Hemangioma of the Alveolar Ridge with Glucose Transporter-1 Reactivity: An Aberrant Presentation. Int J Clin Pediatr Dent 2021;14(2):319-322.

Source of support: Nil

Conflict of interest: None

be a sensitive and specific marker for hemangiomas of the skin, not the mucosa.

This report describes a case of a focal infantile hemangioma presenting on the alveolar ridge of an infant without cutaneous involvement which has not been previously documented. In addition, we describe the importance of GLUT-1 expression as a clinically useful molecular marker in the diagnosis of infantile hemangiomas presenting in the oral cavity. 


\section{Case Description}

An 8.5 kg, 9-month-old female infant was referred by a general dental practitioner to the Department of Pediatric Dentistry for evaluation of a slowly enlarging mass on the patient's maxillary anterior alveolar ridge. The mass had first appeared 15 days after birth and evolved gradually to its present size. The mass bled easily during breastfeeding and the mother was concerned if it was a tumor. There was a history of trauma 1 month back and since spontaneous bleeding was seen from the mass. Parents gave no history of the natal tooth at birth. The infant was first-born by an uncomplicated full-term pregnancy with normal delivery. The patient's medical history was unremarkable and non-contributory.

During the physical examination, no other similar lesions were evident on the skin. Intraoral examination demonstrated a $1 \times 0.5$ $\mathrm{cm}$ pedunculated soft tissue mass on the maxillary alveolar ridge adjacent to the midline. The solitary exophytic mass appeared multilobulated, circumscribed, with a well-defined border overlying primary maxillary left central incisor alveolar mucosa (Fig. 1). The overlying mucosa was reddish-pink and smooth with no ulceration. The mass was firm-rubbery, friable, non-tender, and blanching could not be confirmed due to profuse bleeding on palpation. A presumptive diagnosis of hemangioma was made.

In view of the unprecedented bleeding and to avoid a double visit to the operating room for biopsy, it was decided to perform an excisional biopsy under general anesthesia using electrocautery with complete precautions. The surgical procedure was explained to the parents and informed consent was obtained for the same. After vitamin $\mathrm{K}$ prophylactic injection, the mass was removed en toto using electrocautery, and curettage of the underlying tissue was done. On excision, the incisal edge of the primary maxillary left central incisor was visible.

Microscopic examination of the tissue revealed the presence of abundant dilated capillaries with RBCs and dense mixed inflammatory cell infiltration in the fibrovascular connective suggestive of hemangioma with capillary network and an inflammatory component (Fig. 2A). Immunohistochemical staining was positive for GLUT1 which was detected in the endothelial cells, stromal cells, and microvasculature identified as brown staining (Fig. 2B).

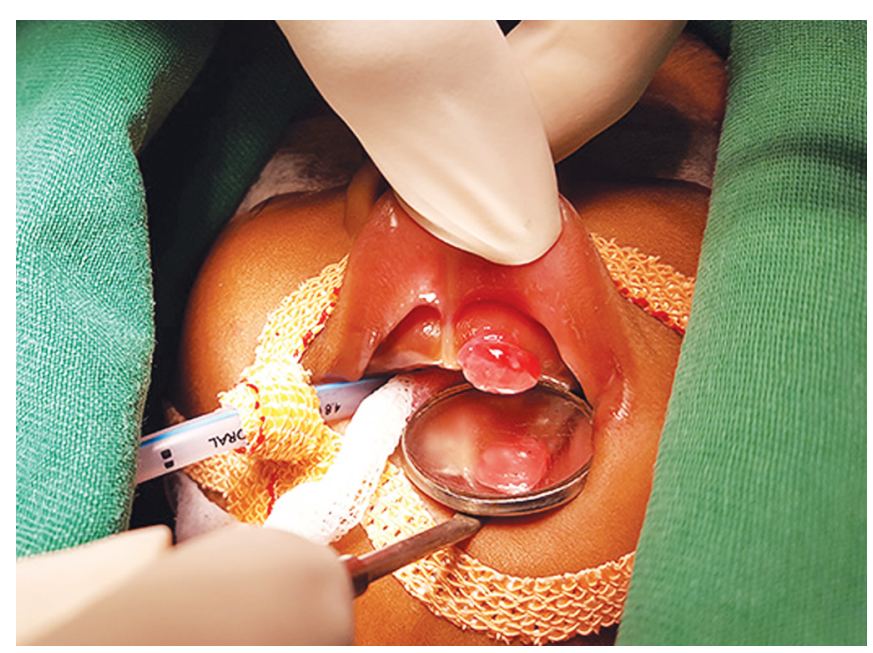

Fig. 1: Solitary pedunculated mass on the maxillary alveolar ridge of a 9-month-old female infant

\section{Discussion}

In 1996, ISSVA created a comprehensive classification scheme based on Mulliken's work on cellular analysis and updated it most recently in 2018. ${ }^{11,12}$ This classification is widely accepted as the gold standard by the numerous medical specialties involved in clinical care and research related to vascular anomalies. Unfortunately, many authors still use imprecise terminology to describe the type of hemangiomas based on the site or histological components and this increased the risk of the patient being treated incorrectly. ${ }^{13}$

Infantile hemangiomas are subclassified as focal, multifocal, segmental, and indeterminate depending on their morphology, extent, or distribution and as superficial, deep, and mixed depending on their location in the skin. ${ }^{12}$ In a study by Waner et al. ${ }^{14}$ of the 232 facial infantile hemangiomas, he observed two patterns of growth of facial hemangiomas, focal lesions that were localized and tumor-like and diffuse segmental lesions were more widespread and plaque like. These focal lesions were postulated due to neural crest-derived mesenchyma of the head at the site of embryonic fusion. It is a rare finding that visceral hemangiomas present without multiple cutaneous lesions. ${ }^{6}$ In the present case, no cutaneous lesions were evident and the lesion was consistent with focal infantile hemangiomas.

The decision on the need to intervene and the therapeutic option should be individualized based on the patient's age, lesion's site and size, clinical features, risks and benefits of the potential intervention available, professional experience, and psychosocial impact on the child and the parent. In the oral cavity, bleeding, ulceration, and infection are the complications that may develop after masticatory minor trauma. ${ }^{3}$ Thomas et al. ${ }^{15}$ reported that infants may experience failure to thrive due to increased metabolic demand from the proliferating tissue, dysphagia from oral or airway obstruction, and feeding problems caused by oral aversion and sensory problems. It is for this reason that authors suggest surgical intervention should be preferred over non-treatment for hemangiomas of the oral cavity unlike active non-intervention for infantile hemangiomas for the skin.

In our case, the patient had several bleeding episodes, the mother had feeding difficulties and hence surgical excision with a minimally invasive approach was used. Electrocautery is ubiquitous in its presence within the operating room as the equipment is less expensive, is more readily available, and requires less training to use than laser equipment. Hence, it served as a feasible treatment option to control trans-operative bleeding for any vascular lesion excision without a prior confirmed diagnosis.

Most infantile hemangiomas could be diagnosed by an accurate history, clinical, and histopathological examination., ${ }^{911}$ Clinically, the present case closely resembled pyogenic granuloma which is a reactive vascular lesion occurring in response to a natal tooth in infants. Another oral lesion that presents at birth with a broad base attached to the alveolar ridge and no spontaneous bleeding and similar predilection for the maxillary alveolar process is congenital epulis. Although rare in the oral cavity, congenital hemangiomas were ruled out as they appear fully grown at birth with no postnatal growth, whereas vascular malformations present at birth, grow commensurately with the patient, and do not spontaneously regress. ${ }^{6,16}$ Our patient presented with a history of spontaneous spurting of blood from the lesion and excessive bleeding on palpation that was difficult to control rather than an indolent ooze suggestive of a vascular lesion like hemangioma. Histologically, late staged hemangiomas could be difficult to differentiate from venous 


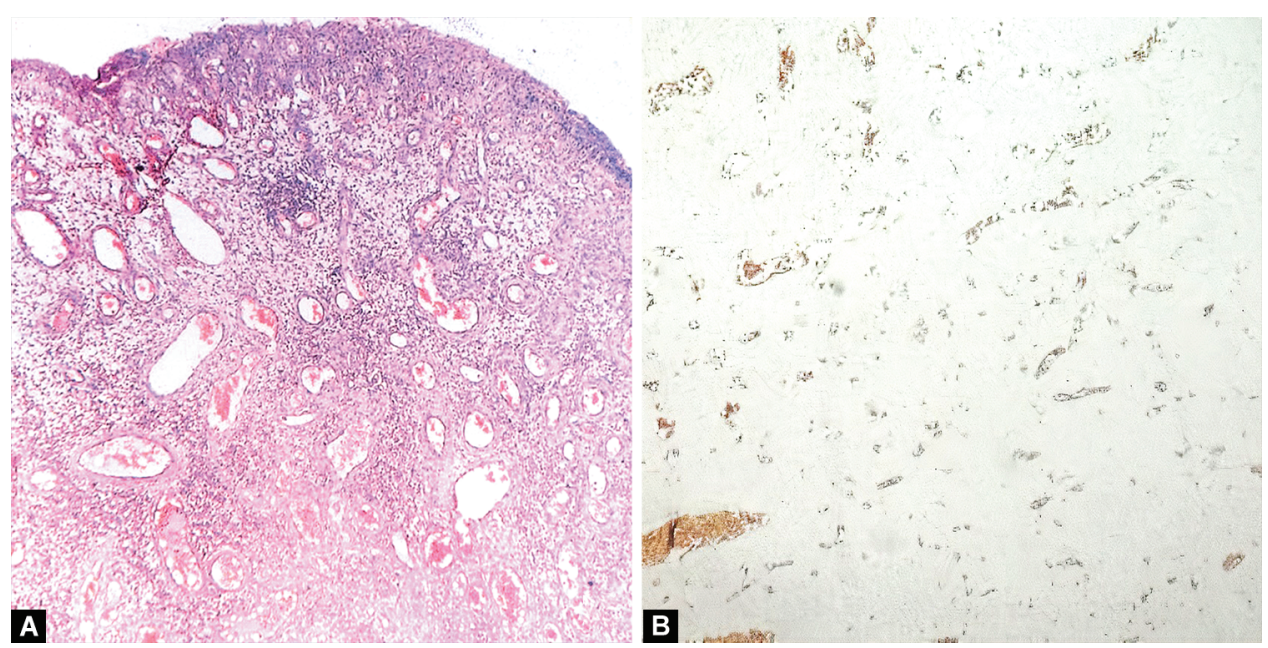

Figs 2A and B: (A) Photomicrograph shows endothelial hyperplasia and connective tissue with endothelium-lined capillaries with erythrocytes and mixed inflammatory cell infiltrates (H\&E staining); (B) Positive staining for GLUT1 marker by endothelial cells of capillaries

or arteriovenous malformations. ${ }^{17}$ Thus, when in a diagnostic dilemma, the use of a distinct biomarker could help distinguish hemangioma from similar lesions.

GLUT1 was reported originally as a marker of infantile hemangiomas of the skin. In IH, the expression of GLUT1 is due to the intrinsic phenotype of the cell types. This is independent of the mitotic activity of the cells unlike in malignancy. ${ }^{16-18}$ Several studies analyzed GLUT1 expression in hemangioma cases diagnosed solely by histology and were reclassified as vascular malformation, pyogenic granuloma, and epithelioid hemangioma. ${ }^{19-21}$ Drut and Drut ${ }^{22}$ studied the expression of GLUT1 in extracutaneous infantile hemangioma occurring in the breast, salivary glands, placenta, liver, and found immunopositivity. The marker was not expressed by congenital non-progressive hemangiomas, vascular and lymphatic malformations, reactive granulation tissue, oral varix, pyogenic granulomas, epithelioid hemangiomas, or hemangioendotheliomas. $3,16,17,20,21,23$ Our case is in agreement with a study done by Johann et al., ${ }^{20}$ which concluded that GLUT1 could be used an auxiliary marker for oral benign vascular lesions.

\section{Conclusion}

The authors advocate the use of GLUT1 marker when there is suspicion of hemangioma for immunohistochemical distinction from other histologically similar vascular lesions. Infants with oral tumors often develop difficulty in feeding, breathing, and lip closure due to the enlarging size of the tumor affecting the nutritional status of the child. Thus, the goal of hemangiomas treatment in infants should reduce or eliminate the tumor, maintain functionality, and esthetics and lessen the psychological distress among the parents with a multidisciplinary team intervention.

\section{Clinical Significance}

Hemangiomas diagnosis is clinically significant especially in infants as it could create a significant risk of intraoperative bleeding and failure to thrive. We opted for electrosurgery as it could cauterize any feeding vessels in addition to removing the tumor. The aim should be minimally invasive surgical management with minimal complications and faster recovery.

\section{References}

1. Winer LH. Hemangiomas; histologic structure, and treatment. Calif Med 1952;77(4):242-247.

2. Corrêa PH, Nunes LC, Johann AC, et al. Prevalence of oral hemangioma, vascular malformation and varix in a Brazilian population. Braz Oral Res 2007;21(1):40-45. DOI: 10.1590/s1806-83242007000100007.

3. Fočo F, Brkić A. Vascular anomalies of the maxillofacial region: Diagnosis and management. In: Motamedi MHK, ed. A Textbook of advanced oral and maxillofacial surgery. Rijeka: IntechOpen; 2013. pp. 303-323. [cited 2020 Aug 05]. Available from: https:// www.intechopen.com/books/atextbook-of-advanced-oral-andmaxillofacial-surgery/vascularanomalies-of-the-maxillofacialregion-diagnosis-and-management.

4. Nanda VS. Management of capillary hemangiomas. West J Med 1994;160(4):364.

5. Amir J, Metzker A, Krikler R, et al. Strawberry hemangioma in preterm infants. Pediatr Dermatol 1986;3(4):331-332. DOI: 10.1111/j.15251470.1986.tb00535.x.

6. Mulliken JB, Burrows PE, Fishman SJ. Mulliken and Young's vascular anomalies: hemangiomas and malformations. 2nd ed., Oxford: Oxford University Press; 2013. pp. 22-110.

7. Sahin G, Duzcan-Kilimci D, Tanyildiz HG. Epidemiological features and risks of hemangiomas. Turk J Pediatr 2017;59(6):664-669. DOI: 10.24953/turkjped.2017.06.007.

8. Finn MC, Glowacki J, Mulliken JB. Congenital vascular lesions: clinical application of a new classification. J Pediatr Surg 1983;18(6):894-900. DOI: 10.1016/s0022-3468(83)80043-8.

9. Acikgoz A, Sakallioglu U, Ozdamar S, et al. Rare benign tumours of oral cavity--capillary haemangioma of palatal mucosa: a case report. Int J Paediatr Dent 2000;10(2):161-165. DOI: 10.1046/j.1365263x.2000.00188.x.

10. Bonet-Coloma C, Minguez-Martinez I, Palma-Carrio C, et al. Clinical characteristics, treatment and outcome of 28 oral haemangiomas in pediatric patients. Med Oral Patol Oral Cir Bucal 2011;16(1):e19-e22. DOI: 10.4317/medoral.16.e19.

11. Mulliken JB, Glowacki J. Hemangiomas and vascular malformations in infants and children: a classification based on endothelial characteristics. Plast Reconstr Surg 1982;69(3):412-422. DOI: 10.1097/00006534-198203000-00002.

12. ISSVA Classification of Vascular Anomalies [Internet]. Milwaukee: International Society for the Study of Vascular Anomalies; 2018 [cited 2020 Aug 05]. Available at: https://www.issva.org/ classification. 
13. Hassanein AH, Mulliken JB, Fishman SJ, et al. Evaluation of terminology for vascular anomalies in current literature. Plast Reconstr Surg 2011;127(1):347-351. DOI: 10.1097/PRS.0b013e3181f95b83.

14. Waner $M$, North $P E$, Scherer $K A$, et al. The nonrandom distribution of facial hemangiomas. Arch Dermatol 2003;139(7):869-875. DOI: 10.1001/archderm.139.7.869.

15. Thomas MW, Burkhart CN, Vaghani SP, et al. Failure to thrive in infants with complicated facial hemangiomas. Pediatr Dermatol 2012;29(1):49-52. DOI: 10.1111/j.1525-1470.2011.01400.x.

16. North PE, Waner M, Mizeracki A, et al. GLUT1: a newly discovered immunohistochemical marker for juvenile hemangiomas. Hum Pathol 2000;31(1):11-22. DOI: 10.1016/s0046-8177(00)80192-6.

17. Leon-Villapalos J, Wolfe K, Kangesu L. GLUT-1: an extra diagnostic tool to differentiate between haemangiomas and vascular malformations. Br J Plast Surg 2005;58(3):348-352. DOI: 10.1016/j.bjps.2004. 05.029 .

18. Huang L, Nakayama $\mathrm{H}$, Klagsbrun $\mathrm{M}$, et al. Glucose transporter 1-positive endothelial cells in infantile hemangioma exhibit features of facultative stem cells. Stem Cells 2015;33(1):133-145. DOI: 10.1002/ stem.1841.

19. de Oliveira DH, da Silveira EJ, de Medeiros AM, et al. Study of the etiopathogenesis and differential diagnosis of oral vascular lesions by immunoexpression of GLUT-1 and HIF-1alpha. J Oral Pathol Med 2014;43(1):76-80. DOI: 10.1111/jop.12092.

20. Johann AC, Salla JT, Gomez RS, et al. GLUT-1 in oral benign vascular lesions. Oral Dis 2007;13(1):51-55. DOI: 10.1111/j.16010825.2006.01246.x.

21. Dyduch G, Okon K, Mierzynski W. Benign vascular proliferations-an immunohistochemical and comparative study. Pol J Pathol 2004;55(2):59-64.

22. Drut RM, Drut R. Extracutaneous infantile haemangioma is also Glut1 positive. J Clin Pathol 2004;57(11):1197-1200. DOI: 10.1136/ jcp.2003.012682.

23. North PE, Waner M, Mizeracki A, et al. A unique microvascular phenotype shared by juvenile hemangiomas and human placenta. Arch Dermatol 2001;137(5):559-570. 